\title{
The remarkable light and colour variability of Small Magellanic Cloud Be stars
}

\author{
W. J. de Wit ${ }^{1}$, H. J. G. L. M. Lamers ${ }^{2,3}$, J. B. Marquette ${ }^{4}$, and J. P. Beaulieu ${ }^{4}$ \\ ${ }^{1}$ Laboratoire d'Astrophysique de Grenoble, Université Joseph Fourier, BP 53, 38041 Grenoble Cedex 9, France \\ e-mail: dewit@obs.ujf-grenoble.fr \\ 2 Astronomical Institute, Utrecht University, Princetonplein 5, 3584 CC Utrecht, The Netherlands \\ 3 SRON Laboratory for Space Research, Sorbonnelaan 2, 3584 CA Utrecht, The Netherlands \\ ${ }^{4}$ Institut d'Astrophysique de Paris, UMR 7095 CNRS, Université Pierre \& Marie Curie, 98bis boulevard Arago, 75014 Paris, France \\ Received 3 March 2006 / Accepted 12 June 2006
}

ABSTRACT

\begin{abstract}
Aims. To test if the irregularly variable optical emission of hot stars in the Small Magellanic Cloud is due to variable amounts of Brehmstrahlung by ionized material in the circumstellar environment. The light curve variability of this sample of hot stars ( 200 objects) is described by Mennickent et al. (2002).

Methods. (1) Probing the relation between flux excess in the optical and in the near-infrared $J$-band using EROS light curves (time span of 5 years) and 2MASS photometry; (2) analyzing the relation between colour and magnitude of the variability by means of an analytical, time-dependent model of emission from ionized material in an outflowing circumstellar disk.

Results. (1) A correlation between optical and near-IR flux excess is found; (2) a bi-valued relation between excess colour and magnitude for $\sim 100$ objects is discovered. It results in a loop structure in a colour-magnitude diagram. Significantly, this loop is traversed in a clockwise sense for $\sim 90 \%$ of the stars, and anti-clockwise for the remainder; (3) a simple analytical model of an outflowing disk and a variable mass loss rate is capable of reproducing observed light curves and the bi-valued colour-magnitude relation.

Conclusions. The optical variability of a large fraction of the hot stars is due to variations in the amount of Brehmstrahlung. The circumstellar envelope can contribute up to $50 \%$ of the total optical flux. This variability can be interpreted as due to an outflowing ionized CS disk, that evolves into a ring structure. The observed bi-valued colour-magnitude relation is an optical depth effect at the various wavelengths of interest. The loop is traversed clockwise by outflowing matter, but anti-clockwise by infalling material. As the material is generally outflowing, the CS environment of the star is progressively cleared once the star stops losing mass. This study makes use of public OGLE, MACHO and 2MASS data.
\end{abstract}

Key words. stars: emission line, Be - stars: mass-loss - stars: circumstellar matter - galaxies: Magellanic Clouds

\section{Introduction}

An important contribution to observational astronomy and in particular to the studies of variable stars was made in the nineties by the microlensing experiments: EROS (e.g. Aubourg et al. 1995), MACHO (e.g. Alcock et al. 1993), OGLE (e.g. Udalski et al. 1997). The projects produced an enormous quantity of photometric data for millions of stars in the Galactic Bulge and the Magellanic Clouds covering nearly a decade of continuous observations. Among the interesting discoveries reported are the Magellanic Clouds' blue variable stars. Two papers in 2002 by Keller et al. (MACHO) and Mennickent et al. (OGLE) reported the huge variety of photometric variability displayed by hundreds of blue stars in the Large and Small Magellanic Cloud, respectively. Both papers type the various light curves according to their morphology. A statistical comparison of various characteristic quantities of the variability between LMC and SMC blue variable stars resulted in pronounced differences for phenomenologically similar light curves (Sabogal et al. 2005). Keller et al. (2002, hereafter K02) examine the presence of Balmer line emission in a subset of their sample and find a detection rate of $91 \%$. They conclude that the Large Magellanic Cloud population of blue variable stars is consistent with the Be star phenomenon (see also Lamers et al. 1999, and de Wit et al. 2003).
In the present work, we focus on the phenomenal optical brightness and colour variability of blue variable stars in the Small Magellanic Cloud. The stars and their light curves were first described by Mennickent et al. (2002, hereafter M02). About $7 \%$ of the SMC Be stars was actually found by M02 to have well-defined periodically varying light curves (see Mennickent et al. 2003). These stars are not considered here. Instead we focus on the irregular photometric variability displayed by the majority of the Be stars on time scales of 1 to 1000 days and amplitudes up to $1^{\mathrm{m}}$. Given that the observed Balmer line emission variability correlates with the photometric variability for similar LMC blue variables (K02) and the fact that generally the stars have redder colours at brighter phases, bound-free and free-free (bf-ff) emission originating in an ionized circumstellar envelope (CSE) is a strong candidate for the observed optical variability. This is generally the case for Galactic stars showing the Be phenomenon. A correlation between near-infrared (near-IR) flux excess and optical flux excess should therefore exist for the SMC Be stars. Such a correlation was already shown to exist for Galactic Be stars by Dachs \& Wamsteker (1982). We proceed therefore by cross-correlating the SMC blue variable stars discovered and catalogued by M02 with the near-IR 2MASS catalogues. 
The paper is organized as follows. In Sect. 2 we specify the way the sample of blue variable SMC stars used in this contribution is defined. We note that in the following we refer to the stars simply by Be stars. In Sect. 3 the presentation of the cross-correlation between 2MASS and the sample stars follows. Then in Sect. 4 we describe the remarkable change in optical colour as a function of the brightness. This behaviour is modeled in Sect. 5. We discuss the results and conclude in Sects. 6 and 7.

\section{Data and completeness of the sample}

To investigate if the optical variability correlates with the near-IR flux, we need to determine (1) the minimum light of each program star, supposedly corresponding to the photospheric emission, and (2) the optical brightness of each object at the epoch of the 2MASS near-IR observation. The type and time scale of the displayed variability requires the longest period of observations for a proper analysis. The light variability of the program stars maybe characterized as erratic and an example is given in Fig. 1.

In this paper we use EROS II microlensing data. They cover a period between 5.5 and 6.5 years, with on average one measurement every three nights. EROS II observed simultaneously in two non-standard passbands using a dichroic to split the beam. These passbands are referred to as $V_{\mathrm{E}}$ and $R_{\mathrm{E}}$, where the "E" stands for EROS. These correspond roughly to Johnson-Cousins $V$ and $I$ band (see e.g. de Wit et al. 2003). In their investigation, M02 used the OGLE II data ${ }^{1}$ that cover about 4 years and fully overlaps in time with the EROS II data (see Fig. 1). In some cases we also inspect the publically available MACHO data, whenever the star's light curve appeared possibly periodic or longer term trends were expected. The MACHO data set provides the longest time line of the three microlensing projects, stretching 7 years. EROS and MACHO data only partially overlap, thus creating the possibility to construct and investigate light curves stretching nearly 10 years. Illustrating the three baselines, we have plotted in Fig. 1 all the available microlensing data for the SMC Be star OGLE 004748.79-730519.4.

The 2MASS All Sky data release (Skrutskie et al. 2006) was explored to obtain the infrared $J H K$ measurements of the 941 irregularly varying SMC Be stars as reported in M02. A crosscorrelation between the two datasets resulted in 392 2MASS matches within a distance of 2 arcsec, requiring that the 2MASS sources have reliable photometric measurements in all three near-IR bands. For these objects, 318 EROS light curve could be extracted. The Julian Dates of the 2MASS observations are between $1033.9205(+2450000)$ and $1821.7956(+2450000)$ and are in principle both covered by the EROS observations, unfortunately however gaps do exist in the EROS coverage. The optical magnitude at 2MASS epoch was determined from the red $R_{\mathrm{E}}$ passband (passband 620-920 nm) observations, allowing a 30day interval around the 2MASS epoch. If more than 1 EROS observation is available in this period, the median of these measurements is taken. The standard deviation of these measurements is then taken as the uncertainty in the determined optical magnitude at 2MASS epoch. In this way, for 239 light curves a correspondence between EROS measurements at the 2MASS epoch is found.

For the large majority of the stars, we are confident that the near-IR 2MASS source actually corresponds to the variable optical source. To confirm this, we searched the optical (BVI bands)

1 Presently only the MACHO and OGLE light curve databases are publically available through the web.

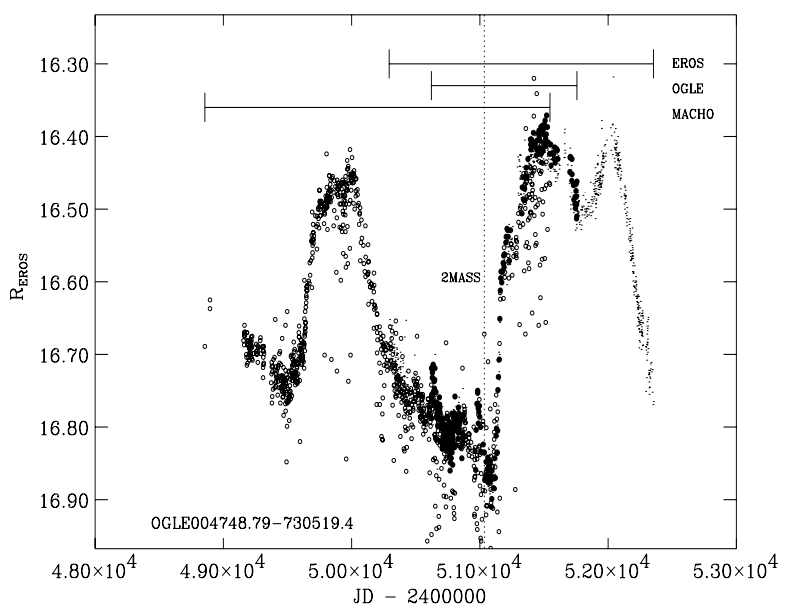

Fig. 1. Light curves produced by three microlensing projects for the Small Magellanic Cloud Be star OGLE 004748.79-730519.4. Open circles are MACHO's red filter $R_{\mathrm{M}}$ data, filled circles are OGLE $I$-band data, small dots are EROS red filter $R_{\mathrm{E}}$ data. We have indicated the covered epoch of each project at the top. The 2MASS epoch of observation is indicated by the vertical dotted line and corresponds here to light minimum. In this particular case, $\Delta R$ (see Fig. 3 ) is nearly zero.

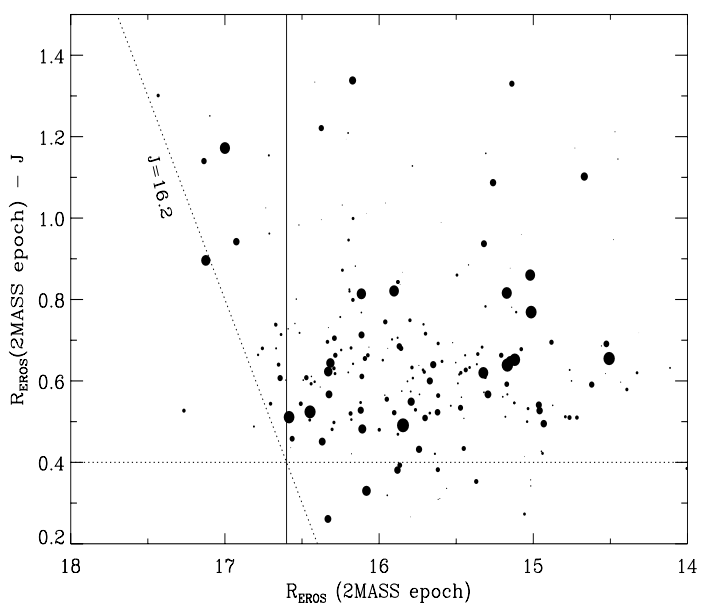

Fig. 2. Completeness of the 2MASS $J$-band measurements for the selected variable stars. Indicated as dotted lines are 2MASS completeness limit and approximately the minimum colour excess of the distribution. Stars brighter than $R_{\mathrm{E}}=16.6^{\mathrm{m}}$ do not suffer from 2MASS incompleteness. Sizes of dots is proportional to the amplitude of optical variability.

OGLE SMC star catalogues (available through CDS), for objects within 2 arcsec of the program stars that have $(V-I)$ colours red enough and $V$-magnitude bright enough to have been picked up by 2MASS $K$-band observations. In 11 cases such a bright red star is present in the OGLE optical SMC star catalogues. We have removed these stars from the sample. With reference to the variability types of $\mathrm{M} 02$, among the selected stars we find 31 of type 1 (luminosity rise and gradual decline), 33 of type 2 (sudden brightness jumps in between periods of near constant flux) and 175 of type 4 (light curves similar to Galactic Be stars).

For all of the light curves we determined the minimum and maximum brightness and the brightness of the object at the 2MASS epoch of observations. The minimum brightness is assumed to correspond to the photosphere of the Be star. The photospheres cover a $R_{\mathrm{E}}$ magnitude range between 13.9-17.6. For the distance of the SMC, these magnitudes would correspond to stars with B spectral types earlier than B 5V approximately. The near-IR excess emission flux is traced by the colour excess 
defined as $R_{\mathrm{E}}^{\min }-J$, where the optical magnitude corresponds to minimum brightness. Given that $R_{\mathrm{E}}$ is close to Johnson $I$-band, we estimate typical photospheric $J$-band magnitudes of the target stars to be between 14.2-18.1, as for B-type photospheres one expects $I_{\mathrm{C}}-J$ to be between -0.5 and -0.25 . This $J$-band magnitude interval actually brackets the 2MASS $J$-band completeness limit of $\sim 16.2^{\mathrm{m}}$. This notwithstanding, the data is not biased as is shown in Fig. 2. Here the distribution of near-IR excess is plotted as a function of the EROS magnitude at 2MASS epoch. The size of each symbol is proportional to the peak-topeak amplitude of the optical variability.

The important thing to notice is that the excess colour is distributed above a value of 0.4 in $\left(R_{\mathrm{E}}-J\right)$, independent of optical magnitude. This indicates that all the stars in our sample have excess colour, which is the main reason why the sample is not biased. The 2MASS completeness only becomes a severe effect at optical magnitudes fainter than $\sim 16.6^{\mathrm{m}}$. In the following, we thus only consider stars brighter than this limit.

\section{Flux excess in the optical and near-IR}

The relation at 2 MASS epoch between the infrared excess and the optical flux excess for all 239 program stars is plotted in Fig. 3. The infrared excess is evaluated by the $\left(R_{\mathrm{E}}^{\min }-J\right)$ colour and the optical flux excess by $\Delta R=\left(R_{\mathrm{E}}^{\mathrm{min}}-R_{\mathrm{E}}\right)$, in which $R_{\mathrm{E}}^{\text {min }}$ is the observed minimum brightness of the EROS II light curve over the 5 years of observations. The size of the symbols corresponds to the $R_{\mathrm{E}}$-band amplitude of variability during the period of EROS II observations. The figure shows that the stars are distributed on an increasingly wider range of $\Delta R$ with increasing near-IR excess. This suggests that the maximum amplitude of optical variability a sample star can display is related to the near-IR excess.

Figure 3 also shows low amplitude stars with large near-IR excess. If indeed these are variable Be stars then their variability time scale should be longer than the 5 years of continuous EROS observations. In that case the underlying B-type photosphere has not been observed and the stars should have optical colours redder than typical B-type star colours. This is indeed seen in the colour-colour diagram of Fig. 4, relating the near-IR excess with the optical colour at minimum brightness. The size of the symbol is again proportional to the amplitude of variability. The figure shows that the low amplitude stars (small symbols) are proportionally redder in the optical $(V-R)_{\mathrm{E}}$ at larger near-IR-excess. On the contrary, the large amplitude stars (large symbols) have generally blue optical colours. It indicates that for the latter stars the photosphere has been observed and thus that $\Delta R$ in Fig. 3 is a correct estimate for the optical flux excess at 2MASS epoch. In Fig. 5 we again plot therefore the same relation as in Fig. 3, but only for those stars with colours $(V-R)_{\mathrm{E}}<-0.2$ at brightness minimum. The relation between the quantities confirms that the optical irregular variability on observed time scales of 1 to 1500 days is correlated with the excess flux in the near-IR.

Given the correlations found by K02 between Balmer line emission and variability and that the variability induces redder colours at brighter phases, our finding here corroborates the notion that the optical variability on time scales of days to years is probably best interpreted as variations of the CSE. The observed maximum amplitude of variability in the optical EROS II $R$-band amounts to $0.9^{\mathrm{m}}$, indicating that at peak flux the CSE can contribute about $50 \%$ to the total optical flux. The distribution of the points in Figs. 3 and 4 can be attributed to variability time scales longer than the 5 year period of EROS observations. This

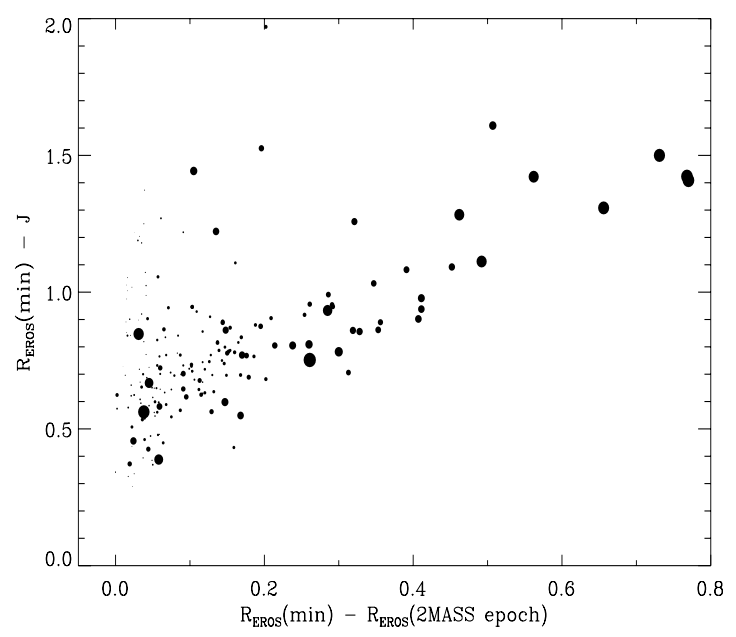

Fig. 3. The relation between flux excess in the near-IR and the flux excess in the optical $(\Delta R)$ at 2MASS epoch for all selected 239 SMC Be stars. The size of the symbol is proportional to the photometric amplitude of the variability measured in the $R_{\mathrm{E}}$ passband. The amplitude of variability ranges from 0 to $\sim 0.9^{\mathrm{m}}$.

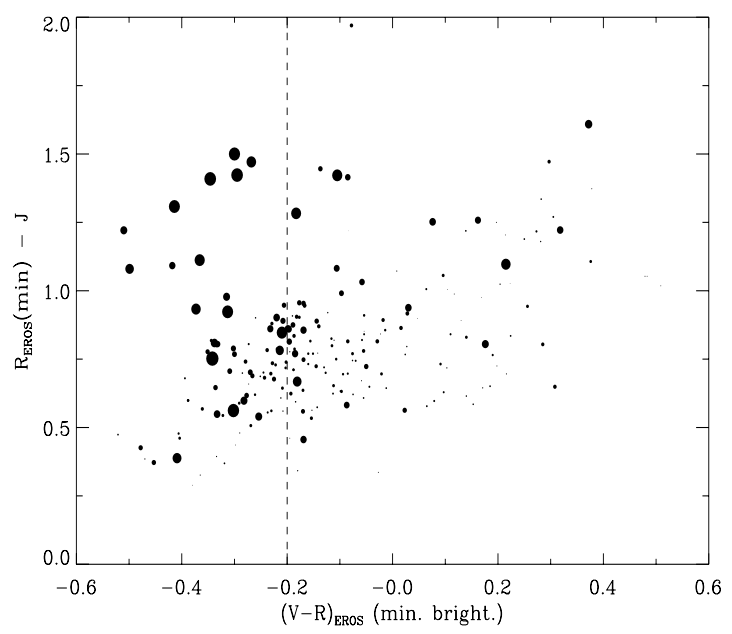

Fig. 4. The relation between optical colour at minimum brightness of the light curve and the near-IR flux excess for all selected SMC Be stars. The size of the symbol is proportional to the photometric amplitude of variability in the EROS light curve. Generally the small amplitude stars have redder optical colours when they have larger near-IR excess. Stars bluer than -0.2 are represented in Fig. 5 .

long time scale has inhibited observations of the B-type photosphere of a fraction of the stars, and hence underestimated the optical flux excess $(\Delta R)$ at the 2MASS epoch of observations. Such a persistent CSE for the low-amplitude stars increases the probability for these stars to be persistent $\mathrm{H} \alpha$ emitters. Crosscorrelating all the Be stars in our sample with the catalogues of SMC H $\alpha$ emitters by Meyssonnier \& Azzopardi (1993) we find a detection rate of $47 \%$. The same percentage of $\mathrm{H} \alpha$ emitters we obtain for the subsample of low-amplitude Be stars, not indicating an increased persistence for this subgroup.

\section{Bi-valued colour-magnitude variation}

The optical colours of Galactic Be stars are observed to become redder when the star increases in brightness for the majority of the long-term variable Be stars (e.g. Moujtahid et al. 1999; Percy \& Bakos 2001). Be star models viewed pole-on by Poeckert \& Marlborough (1978) produce this colour-magnitude relation by 


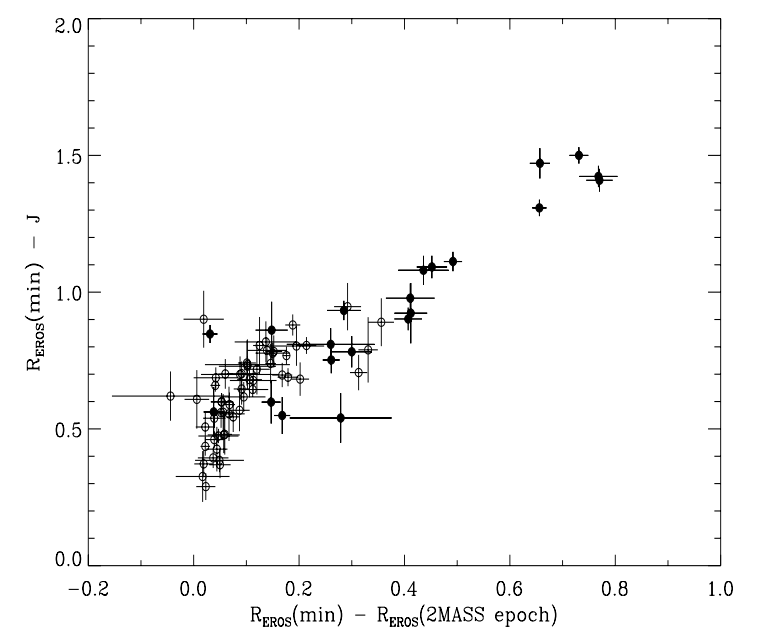

Fig. 5. The relation between flux excess in the near-IR and the flux excess in the optical at the 2MASS epoch of observation. Presented are all stars with an optical colour less than -0.2 at brightness minimum. This secures that the EROS light curves has covered the epoch when the Be star was without CSE. Open symbols have an amplitude of variability less than 0.4 .

scaling the CSE density for a constant outer radius. The change in density leads to a change in the emerging bound-free and freefree flux emission as long as the emission is optically thin. This approach predicts a single valued relation between colour and magnitude for the B-type star and its varying CSE. The opposite trend, i.e. redder optical colour with decreasing brightness, is observed but only in a limited number of Galactic Be stars that are seen under a high inclination (see e.g. Hirata 1982). The result of the previous section secures that the optical variability of many of the SMC blue variable stars is due to ionized material most likely situated in the star's circumstellar environment. In this section we explore what EROS light curves reveal with respect to the change in colour as function of the brightness.

The two panels of Fig. 6 show the light curve and corresponding colour-magnitude relation of a typical and exemplary variability of one blue variable SMC star. The colour-magnitude relation is not single valued but double valued, causing a looplike relation, and is in contrast with what may be expected. Zero values in magnitude and colour correspond to the epoch when minimum flux is observed, presumably the photospheric flux. The object seems to have completed a full variability cycle in about 2 years. Fraction wise, some $40 \%$ of the Be stars in our sample that have an amplitude of variability larger than $0.2^{\mathrm{m}}$ in the $R_{\mathrm{E}}$-band (101 objects in our sample) trace out a loop, either during a flux "burst" or a flux "dip". We have chosen this particular star as an instructive example for the type of colourmagnitude variability observed among the SMC Be stars.

One can subdivide the observed light and colour curve in four phases (see Fig. 6), using the Roman numerals I to IV. As shown by e.g. Sabogal et al. (2005), some $80 \%$ of the Magellanic Cloud blue variables have a shorter flux rising time (phases I and II combined) than a flux decay time (phases III and IV).

- Phase I: the initialization phase. It generally corresponds to a relatively quick increase in both red and blue flux, causing a redder colour.

- Phase II: the saturation phase. The flux increase slows down significantly.

- Phase III: the turn-over phase. Both the blue and red flux decline, but the blue flux does so faster than the red flux, giving rise to a opposite trend between flux and colour, i.e. redder when fainter.

- Phase IV: the decline phase. The flux decreases and the colour becomes bluer.

Interestingly and importantly, these four phases are nearly always observed to be completed in a clock-wise sense! For 9 instances $(10 \%)$ in the subset of the sample with an amplitude larger than $0.2^{\mathrm{m}}$ we find a loop like feature that is completed in a counter clock-wise sense, which we'll discuss in section Sect. 6. Examples of this loop behaviour are illustrated in Fig. 7. The data in each panel is presented in the same way as in Fig. 6, with the exception that the EROS light curve is now shown as an inset in the upper left corner of each panel. The arrow in each panel indicates the time consecutive measurements. The first two rows of Fig. 7 present SMC Be stars that trace out loop structures in a clock-wise sence, for various light curve shapes. The first row shows additional examples of the variability quite similar to the one of Figs. 1 and 6. These are the so-called bumper stars first reported by the MACHO collaboration (Cook et al. 1995). On time scales of years, these Be stars go through a cycle of flux outburst and subsequent decline back to the presumed photospheric emission. An exception to the shape of Fig. 6 is presented in panel b where phases I and IV in the CMD partially coincide. The excess flux of Be stars that vary on somewhat shorter time scale are presented in panel $\mathrm{d}$ and $\mathrm{e}$ of the second row. In panel $\mathrm{f}$ of Fig. 7 actual flux decrease and subsequent recovery to the initial flux level also give rise to two distinct branches in a CMD. In panel $\mathrm{g}$, an example is shown of loop traversed counter clock-wise.

Finally, other types of colour-magnitude variability observed among the full sample are illustrated in panels $h$ and $i$ of the third row in Fig. 7. In these cases there is no indication whatsoever of a loop-like structure, and a redder when fainter trend is observed (panel h). In the last example we show the complicated structure of panel i, where for any given colour there can exist as many as three corresponding magnitudes. For now we refrain from defining various classes or types of colour-magnitude variability. We conclude with the observation that a bi-valued (looplike) colour-magnitude relation seems however a common photometric phenomenon present among Be stars.

\section{Modeling of the light and colour variations}

The dynamics of Be star disks have been proposed to be viscously dominated, transporting angular momentum and material outward (Lee et al. 1991; Porter 1999). The kinematics are observed to be consistent with quasi-Keplerian motion (Dachs et al. 1986). Long-term brightness changes can be understood in terms of an optically thick pseudo photosphere to a more extended and optically thin envelope (Harmanec 1998). The disk loss (transition from Be to B-type star) may occur from the inside out thus creating a ring e.g. in $o$ And (Clark et al. 2003). Ring-like structures are also indicated by the spectroscopic variability of other Galactic Be stars (Rivinius et al. 2001). Below we analyze analytically the effect on the observed excess emission from emitting ionized material in an outflowing circumstellar disk. In our simple model the disk is viewed pole-on (in disks viewed under an angle, the vertical optical depth will increase by a factor $\left.(\sin i)^{-1}\right)$. Inside-out removal of the disk occurs logically, once the stars stops losing mass. 


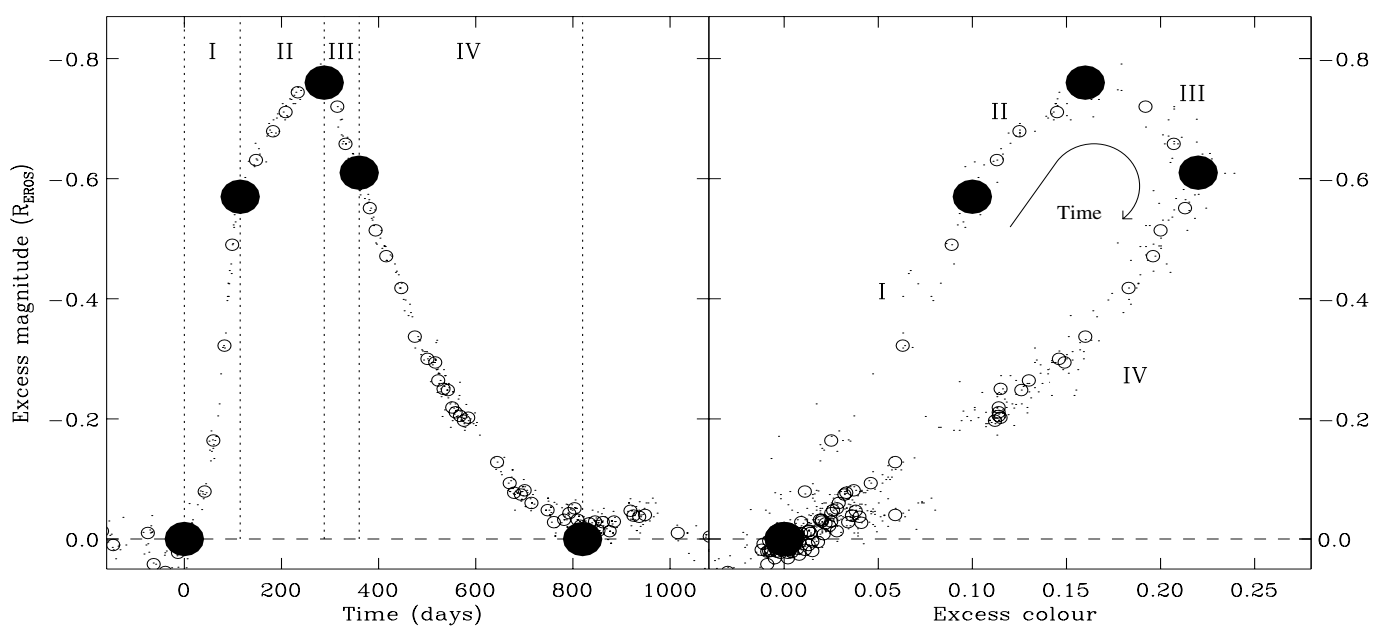

Fig. 6. Light curve (left panel) and CMD (right panel) of the variation of the star OGLE 005209.92-731820.4. Excess magnitude and excess colour are the observed values minus the magnitude and colour at time $=0$ days, i.e. the beginning of the flux increase. The small dots represent all measurements, the open circles are median values in intervals of ten consecutive measurements. The large black dots mark four specific phases. The excess magnitude temporal behaviour produces a loop-like track in the CMD that is traversed in a clock-wise sense.
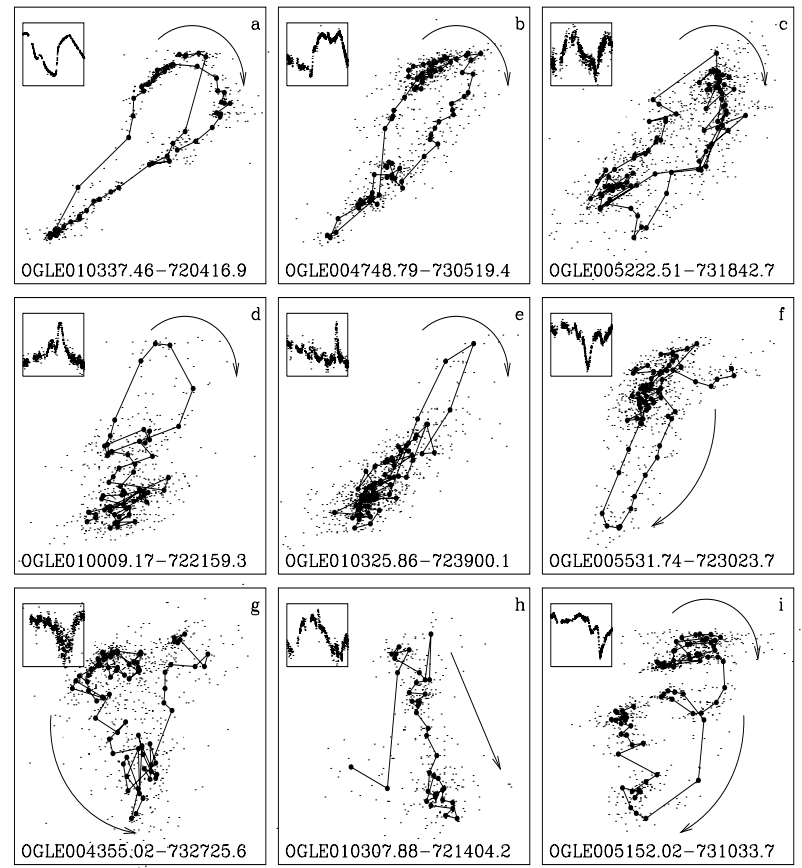

Fig. 7. Some examples of observed CMDs of SMC Be stars, magnitude on $y$-axis and colour on $x$-axis. Corresponding ligh curves are in the inset of each panel. The small and large dots have the same meaning as in Fig. 6. The arrows indicate the direction of time. When a loop is observed (101 cases in our sample) then in $90 \%$ it is traversed clockwise.

\subsection{An analytical description of the variability}

One can solve analytically for the emerging bf-ff flux emission in a time-dependent, outflowing disk (ring) structure by applying a geometrically thin disk approximation (i.e. no vertical structure), which for simplicity is described only by power laws in temperature, velocity, and opening angle

$T=T_{0} x^{-q}, \quad v=v_{0} x^{+p}, \quad \alpha=\alpha_{0} x^{+m}$,

in which $x=r / R_{*}$. By applying mass continuity with respect to the mass loss of the B star, it follows that the density stratification of the disk is then given by $\rho=\rho_{0} x^{n}$, in which the power $n$ equals $-p-m-2$ and $\rho_{0}=\dot{M}_{*} / 2 \pi R_{*}^{2} \alpha_{0} v_{0}$. The absorption coefficients for free-free and bound-free processes which are proportional to $\lambda^{2}$ and depend in the same way on the local values of the physical quantities, and thus a combined expression can be used for their description as given by Brussaard \& van de Hulst (1962). In the Rayleigh-Jeans limit, one can arrive at an analytical expression for the disk emission by dividing the disk in an optically thick and optically thin part. For the $\tau>1$ part one finds the expression

$\frac{F_{\lambda}}{2 \pi R_{*}^{2}}=\frac{c_{1} T_{0} \lambda^{-4}}{-q+2}\left\{x_{\tau=1}^{-q+2}-x_{\text {in }}^{-q+2}\right\}$,

in which $c_{1}=2 k_{b} c$. For $\tau<1$ part of the disk

$\frac{F_{\lambda}}{2 \pi R_{*}^{2}}=\frac{\epsilon_{0} h_{0} \lambda^{-2}}{0.5 q-2 p-m-1}\left\{x_{\text {out }}^{0.5 q-2 p-m-1}-x_{\tau=1}^{0.5 q-2 p-m-1}\right\}$,

in which $\epsilon_{0} h_{0}$ is the product of the emissivity and height of the disk at the stellar surface. The factor $\epsilon_{0}$ is given by $c_{\mathrm{ff}} T^{-0.5} \rho_{0}^{2} c_{1}$, and $h_{0}=R_{*} \alpha_{0}$. The $c_{\mathrm{ff}}$ follows from the absorption coefficient for bound-free and free-free processes, see e.g. Eq. (1) of Lamers $\&$ Waters (1984). At any wavelength, the inner part of the disk is more optically thick (in the vertical direction) than the outer part, because of its $\rho^{2}$ dependence. Similarly, and at any distance $x$ from the star, the optical depth is larger at long wavelength than at short wavelength, because of its $\lambda^{2}$ dependence. So for any wavelength, we can split the disk into an optically thick ring close to the star (if the mass loss rate is high enough) and an optically thin ring at larger distance. Having split the emerging flux in two parts, one has to find the dividing radius for which the vertical optical depth $\tau=1$. This can be done by solving the appropriate power law expression for the optical depth stratification

$x_{\tau=1}^{\lambda}=\left(\tau_{0}^{\lambda} \lambda^{2}\right)^{\frac{-1}{1.5 q-2 p-m-3}}$.

The $\tau_{0}$ in this equation is determined from the absorption coefficient for bf-ff emission, the density, the temperature, and the opening angle, and is given by $\tau_{0}^{\lambda}=c_{\mathrm{ff}} T^{-0.5} \rho_{0}^{2} h_{0}$. It represents the vertical optical depth of the disk at the distance $x=1$. Finally the above geometry can be made time dependent by switching on and off the star's mass loss and solving for the inner and outer rim of the disk given the power law relation for the outflow velocity (Eq. (1)). 
The optically thick part (Eq. (2)) is governed by $q$ value of the temperature structure of the disk as expected. The $\lambda^{-4}$ dependence is a consequence of the applied Rayleigh-Jeans approximation. The optically thin part is governed by the combined action of the emissivity and optical depth, determined by the three physical quantities used to approximate the disk structure, i.e. temperature, radial velocity and opening angle. The optically thin part has a $\lambda^{-2}$ dependence. The equations describing optically thin and thick emission thus depend in a different way on the wavelength, i.e. they have different spectral slopes and thus different colours. The key to the bi-valued colour-magnitude behaviour is a transition from an optically thick to an optically thin regime (or vice versa).

\subsection{A simple model for the prediction of variable excess radiation}

A calculation using this formalism is presented in Fig. 8. The figure shows the logarithm of the emerging disk flux at $\lambda=6475 \AA$ against the colour. The colour is with respect to the emerging disk flux at $4730 \AA$. These wavelengths correspond to the effective wavelength of the EROS dichroics for hot stars. We emphasize that pure disk emission is plotted, without the stellar radiation.

The central star has an effective temperature of $40000 \mathrm{~K}$ and a radius of $R_{*}=7.0 R_{\odot}$. The temperature of the disk $\left(T_{0}\right.$ in Eq. (2)) is chosen as $80 \%$ of the stellar effective temperature, i.e. $32000 \mathrm{~K}$ and it is chosen to be constant through the disk. In this example, also the radial outflow velocity of $1.0 \mathrm{~km} \mathrm{~s}^{-1}$, and the opening angle of $5^{\circ}$ are constants, and thus we have $q=p=m=0$. The rather high stellar (and thus disk) temperature is required to comply with the Rayleigh-Jeans approximation used in the derivation of Eqs. (2) and (3). The disk is produced by a star with a mass-loss rate of $10^{-7} M_{\odot} \mathrm{yr}^{-1}$. The star continues to lose mass for a period of one year at this constant rate. This correspond to phases labelled I and II in the CMD of Fig. 8. During this year, the density of the disk at the stellar surface is always $4.8 \times 10^{-10} \mathrm{~g} \mathrm{~cm}^{-3}$. Initially (phase I) the disk is optically thick in both the blue and red pass band $(\tau=21$ and $\tau=44$ respectively). The observed colour of the system is in this case independent of any disk parameter and only determined by the effective wavelength of the photometric pass-band, as can be verified from Eq. (2). The colour of the disk does not change, but the total emerging emission continues to rise due to the increasing disk radius.

The colour of the disk starts to change in phase II, when the disk densities at the outer disk edge have attained values for which the blue radiation is optically thin. After sometime also the red radiation becomes optically thin at the outer disk radius. At the end of phase II, the change in colour and magnitude for each consecutive time step becomes progressively smaller, asymptotically reaching the colour and emission of an infinitely extended disk. This corresponds to the peak value of the excess flux. At the beginning of phase III the mass loss is stopped and the disk is cleared from inside out. Phases II and III constitute a transition phase between a fully optically thick disk and a fully optically thin disk. The disk consists now at both wavelengths of an optically thick and optically thin part. In the blue passband the optically thick disk stretches out to $2.7 R_{*}$ and the red passband emission is optically thick out to $3.5 R_{*}$. The disk has a physical size of $\sim 7.4 R_{*}$. At the outer edge of the disk the optical depths are 0.05 and 0.1 for the blue and red passband respectively. The termination of the mass loss at phase III causes the inner

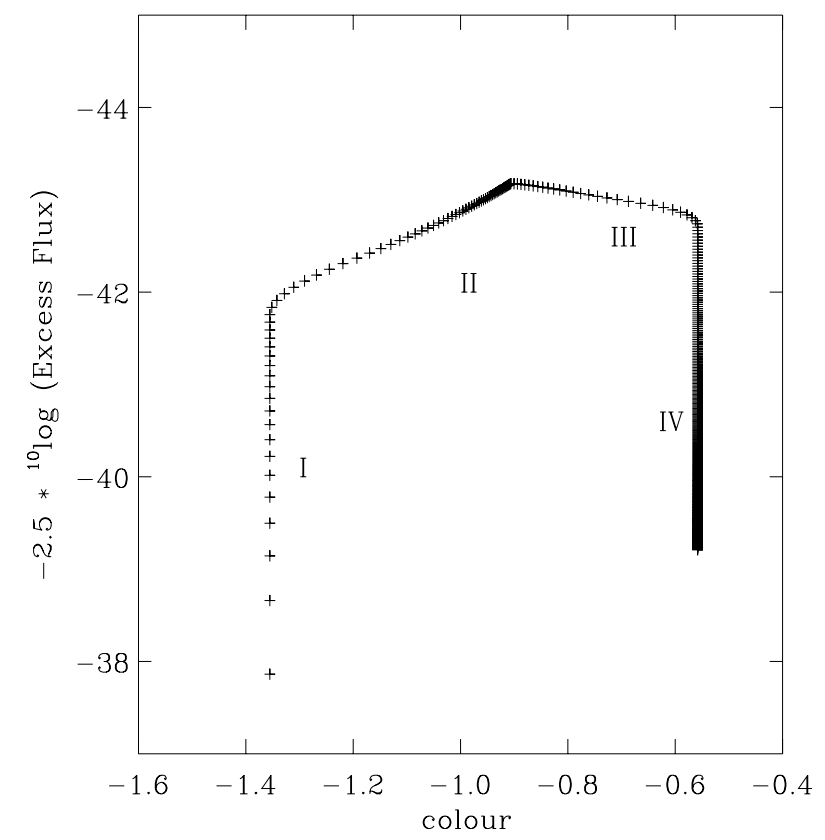

Fig. 8. Emission from the disk only, as calculated using Eqs. (1)-(4) for wavelengths $4730 \AA$ and $6475 \AA$. The star loses mass (phases I and II) at a rate of $10^{-7} M_{\odot} \mathrm{yr}^{-1}$ for 1 full year. During phases III and IV the disk is cleared from the inside out. Crosses mark the evolution every 5 days. The disk is characterized by $T_{0}=32000 \mathrm{~K}, v_{0}=1.0 \mathrm{~km} \mathrm{~s}^{-1}$, and $\alpha_{0}=5^{\circ}$. Temperature, opening angle and radial velocity of the disk are constants during all four phases $(q=p=m=0)$.

optically thick parts of the disk to be removed from the inside out, the flux in the red pass-band to drop, and the overall colour of the disk to approach the colour of the optically thin disk. At phase IV the optically thick part of the disk has been completely cleared and the colour corresponds to the ratio of the blue to red flux in a fully optically thin disk, and determined by Eq. (3). A ring structure continues to diminish in flux at constant colour, as the density continues to decrease due to the expansion.

\subsection{An example of modeled variability}

Three illustrations of the model now including a stellar contribution are presented in the panels of Fig. 9. The calculated fluxes are compared to observations of three burst events observed in three different Be stars (small dots). The fluxes calculation here is a slight modification to the analytical model of the previous section. Now the flux is calculated applying the correct optical depth at each radius, and not the approximation that the disk consists of a pure optically thick and a pure optically thin part. Also, now we use the proper expression for the Planck function, and thus we are no longer restricted to the Rayleigh-Jeans tail of the spectrum. The left column corresponds to the light curves and the right column to the corresponding CMDs. In both cases the $Y$ ordinate is chosen to present the -2.5 times the logarithm of the monochromatic flux ratio $Z_{\lambda}=F_{\lambda} / F_{\lambda}^{*}$ as introduced by Lamers \& Waters (1984). The disk temperature at the stellar surface is chosen to be 0.8 that of the star. For the stars in the sample there is no spectral information available yet, and we chose therefore a stellar effective temperature and radius corresponding to the most likely spectral type for the Be stars in the OGLE sample, i.e. B 2 (see Mennickent et al. 2002). The CMDs of the right-hand side panels have as $X$-ordinate the ratio of the two monochromatic flux ratios corresponding to the effective 

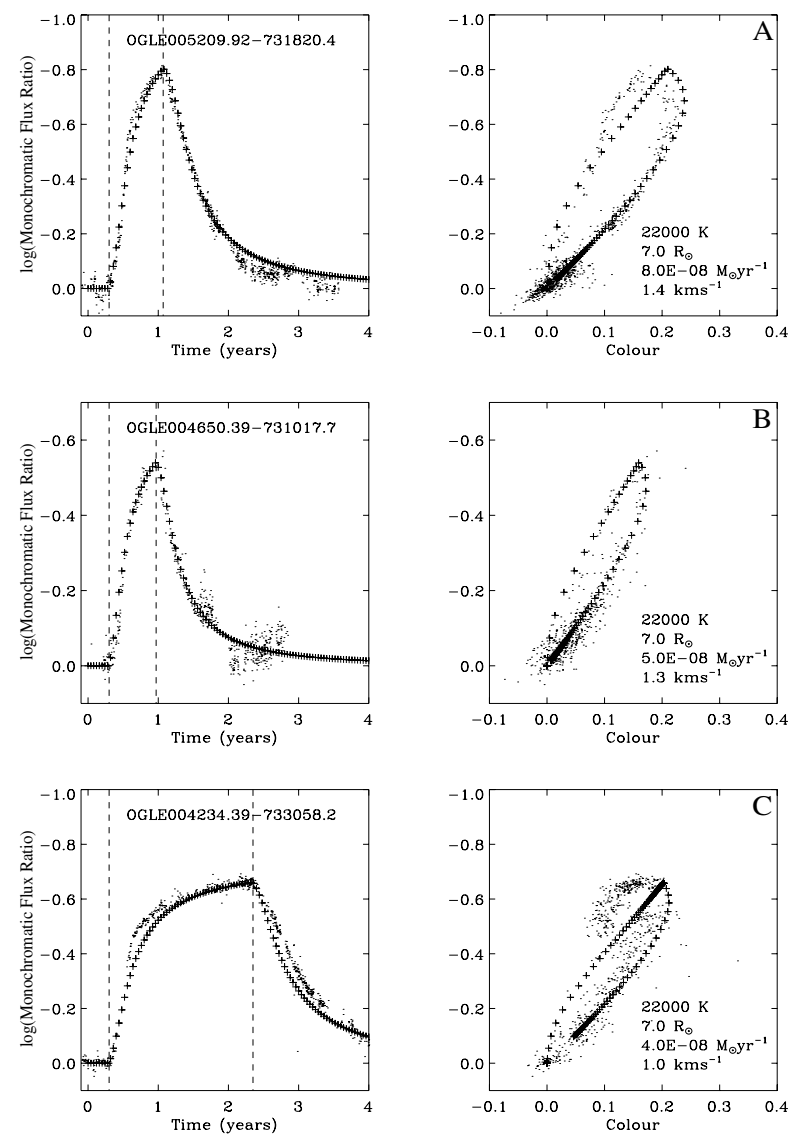

Fig. 9. Model fits to eruption events of three different SMC Be star (as small dots). The left column are calculated light curves of magnitude against time in years. The right column presents the corresponding calculated CMD. The parameters of the fit are also given. Temperature, velocity and opening angle of the disk are constants. The two dashed lines in the left column indicate when the central star was losing mass. See text for further explanation of the differences.

wavelengths of the EROS blue and red filters. The period in which the central star loses mass is marked by the two vertical dashed lines, and is different for each burst event.

The shape of the light curves are quite similar and we briefly describe this. The central B-type star loses mass at a rate of a few times $10^{-8} M_{\odot} \mathrm{yr}^{-1}$. The material flows out a very modest radial velocity of about a $1.0 \mathrm{~km} \mathrm{~s}^{-1}$. During the star's mass losing period, the excess flux rises steeply as the disk grows from the stellar surface outward. The colour hardly changes initially, but becomes redder after an magnitude increase of about -0.1 . This corresponds to phases I and the initial part of phase II in Fig. 8. The turnover in colour corresponds to the moment that the blue radiation becomes optically thin at the outer radius. The flux increase starts to level once the ever increasing outer edge of the disk has attained densities for which the red radiation also becomes optically thin. This corresponds to approximately halfway of phase II of Fig. 8. Once the mass loss is turned off, corresponding to maximum light, the excess flux drops instantly and quickly, because the optically thick parts near the stellar surface are the first to disappear; the disk evolves into a ring. The corresponding colour first becomes redder as relatively more optically thick blue flux is removed from the inner edge of the disk (because the red optically thick part is larger than the blue optically thick part). Once all optically thick material (blue and red) is removed from the inner edge of the disk, the colour of the disk follows the track of the optically thin emission. The exact observed relation between the colour and magnitude depends not only on the optical thickness of the disk, but also on the stellar temperature. The reason is that for the optical filters and temperatures of the star, the Rayleigh-Jeans approximation is no longer valid. Thus by chosing a disk temperature of 0.8 times the effective temperature of the central star, one is adding two energy distributions with different spectral slopes.

All three events were fit by varying the mass-loss rate and the outflow velocity of the disk material. The outflow velocity, the temperature and the opening angle of the disk (see Eq. (1)) are for each calculation constant. The two panels of the top row (case A) show the fit to the light curve and the colour-magnitude diagram of the star already presented in Fig. 6. In the three cases the outflow velocity is found to be order $1 \mathrm{~km} \mathrm{~s}^{-1}$, which is set by shape of the light curve during the mass losing period. A higher $v_{0}$ has the effect of (1) making the optically thick part of the disk smaller and (2) reducing the required time to fill this region with material once the star starts losing mass. By increasing the mass loss rate, the time scale for filling in the optically thick part of the disk is the same as for low outflow velocities (obtaining a similar shape of the flux rise of the light curve) but for much larger optically thick disk and thus a larger emerging flux. Thus the shape of the light curve at the onset of the mass loss of the star can be fit only with the quoted combination of outflow velocities and mass loss rates. The disk parameter values quoted in Fig. 9 depend also on the (unknown) stellar radius and temperature, and may change by a factor of 2 .

The relation between colour and magnitude is not reproduced satisfactorily by the model. At peak flux the colour is observed to be bluer in case A and C of Fig. 9. Note that also in this case the calculation does not reproduce the initial colourmagnitude behaviour satisfactorily. The two observed branches are much closer together than the calculated ones. This may indicate the in reality the mass loss of the star slowly sets in, and does not switch abruptly from zero to $10^{-8} M_{\odot} \mathrm{yr}^{-1}$ as was assumed. Alternatively it may also be explained if at the initial stages the material has a higher radial velocity or if the temperature of the disk material is lower.

\section{Discussion}

In this paper we have provided evidence that the optical excess emission of an ensemble of blue variable stars in the Small Magellanic Cloud is correlated with the near-IR excess emission. The sample contains irregular variable stars, and no selection was made regarding their type of irregular variability. It supports the idea that the blue variable SMC stars, as an ensemble, are stars showing the Be phenomenon. The maximum optical excess emission found in this study (nearly $50 \%$ of the continuum) does not seem to be an exceptional feature for Be stars. Galactic Be type stars have been reported to show similar and even larger amplitude optical flux out bursts with $\Delta V>1^{\mathrm{m}}$ (e.g. Dachs \& Wamsteker 1982; Apparao 1991; Mennickent \& Vogt 1991).

The time variable optical excess emission of the SMC blue variable stars show a bi-valued relation between magnitude and colour. Such a relation between these two quantities is reported here for the first time. This relation results in a loop-like track in a CMD. We were able to identify this feature most easily for stars that have flux outbursts, but they are also observed among stars with flux decreases. Using an analytic description for the bound-free and free-free emission of an outflowing disk near a mass-losing B-type star we interpret this photometric effect in terms of a different time-evolution of the optical depth for various wavelengths. The model is phenomenological and is partly 
based on recent observations that Be-star disks evolve into ring structures. A geometrical evolution may occur during the phase of disk dissipation where material becomes increasingly optically thin from the inside out (e.g. Rivinius et al. 2001). This may indicate that either material is falling back on the star or flows out. In the case of the late-type Galactic Be star $o$ And the disk seems to be lost from the inside out, but also appears to be rebuilt in a similar manner, arguing for an outflowing nature of the material (Clark et al. 2003). In our description the only timevariable parameter is the mass-loss rate of the central star, that was approximated by only two states (on, off). Such a mass-loss pattern may be expected if for instance the disk is due to nonradial pulsations of the central star (e.g. Rivinius et al. 1998). Our approach allows us to produce a bi-valued relation between colour and magnitude, that can fit reasonably well the observed data for objects that show flux outbursts on time scales between 0.5 and 2 years. In this scenario flux decreases can be interpreted as a temporarily halt to the mass-loss of the central star, causing the inner optically thick region to be evacuated.

The colours of Be stars are expected to change continuously, as soon as the star enters in a mass-losing phase. In only one scenario (within the framework of the model discussed here) a constant excess colour is observed and that is during longlived, constant mass-losing periods in which the circumstellar disk asymptotically approaches the colour and the excess emission of an infinite disk. A single relation between colour and magnitude is expected if the disk material remains optically thin. Multi-valued relation are observed when the circumstellar environment consists of one or multiple rings.

The evolution of photometric colours are different from that of static models and may allow to explain also "anomalous" near-IR colours as reported in Dougherty et al. (1994), that could not be accounted for using the static disk model of Waters (1986), but can however be reproduced using an outflowing disk model. This is shown in Fig. 10, in which we reproduce Fig. 8d of Dougherty et al. together with the evolution of near-IR colours of an outflowing disk/ring structure. As noted in Dougherty et al. the four stars indicated by their names have in particular deviant near-IR colours. These colour are however expected during the phase of disk build-up. The few stars found in this region is consistent with the idea that disk creation takes generally much less time than the disk dispersal.

The bi-valued relation between colour and magnitude results in a loop-like structure in a CMD. In those cases in the sample for which the amplitude of the photometric variability is larger than $0.2^{\mathrm{m}}$ we could identify a loop in $40 \%$ of the stars. Among these 101 objects the loop is traversed in a clock-wise sense with time for $90 \%$ of the cases. This fact conveys important information on the physical direction the emitting material is going. Outflowing material traverses the loop clock-wise, whereas accreting material traverses the loop counter clock-wise. Simple multi-epoch photometry at two different passbands can thus establish whether the star is losing or accreting material. In case of classical Be stars, it can be established whether the central star reaccretes (part of) its previously expelled material. If the disk is (partially) supported by radiation then loss of radiative support will lead naturally to an accretion phase where the disc falls back on to the star (e.g. Porter 1999). In our sample of Be stars the re-accretion of matter, using the loop diagnostic occurs in 10 cases only. This constitutes some $10 \%$ of the stars showing loops. Thus in general the material in the CS of Be stars (at least in the SMC) acquires enough angular momentum so as to escape the system altogether, but the data thus also shows that Be stars in some instances actually accrete material. Whether

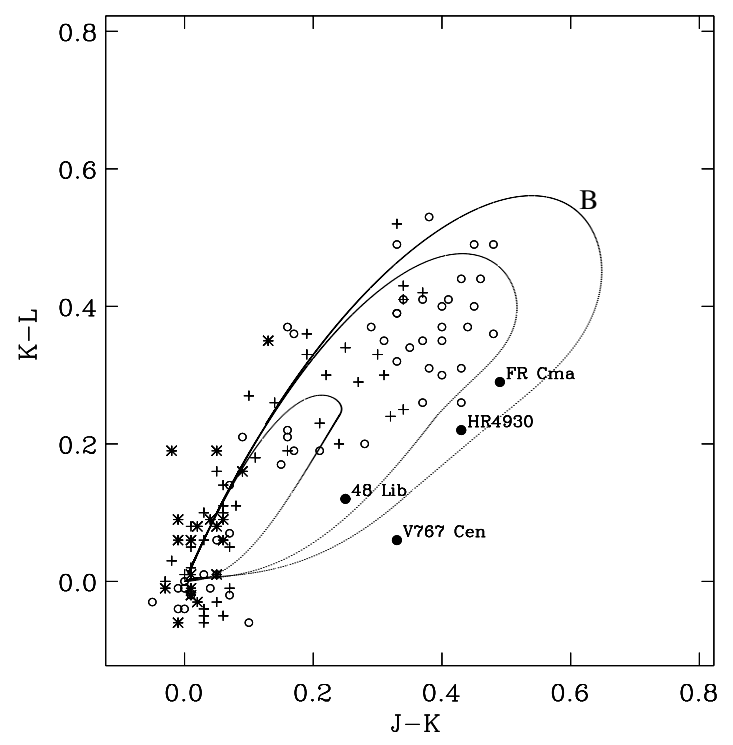

Fig. 10. The evolution of the near infrared colours for case B of Fig. 9 (outer track), and two cases with a smaller mass loss rate, $3 \times 10^{-8}$ and $1 \times 10^{-8} M_{\odot} \mathrm{yr}^{-1}$. The tracks are traversed in a counter clock-wise sense. Symbols represent data taken from Dougherty et al. (1991) for early (circles) mid (crosses) and late-type (asterisks) Galactic Be stars. During disk creation, one can expect quite blue $(K-L)$ colours at given $(J-K)$, as observed for some stars (4 most extreme cases are indicated, following Dougherty et al. 1994).

these stars are actually classical Be stars needs to be confirmed. Loop structures in CMDs may thus also be observed in accreting young stellar objects, in which case the material that is being accreted changes from optically thin to thick, traversing the loop counter clock-wise.

\section{Conclusions}

Irregular optical variability observed in a large ensemble of blue stars in the Small Magellanic Cloud is due to variations in the amount of Brehmstrahlung, most likely located in a circumstellar envelope or disk in analogy with classical Be stars. The CS disk can thus contribute up to $50 \%$ of the total optical luminosity of the stars, as derived from the amplitude of optical variability. Using a simple model we have shown that these flux changes can be interpreted as due to a time variable mass loss of the central star. The disk material is always outflowing. The outflowing material is observed to produce a bi-valued colour-magnitude relation (a loop in a colour-magnitude diagram), that can be ascribed to optical depth effects. The loop is traversed in a clockwise sense for outflowing material, but in a anti clock-wise sense for accreted material. The data is compatible with disks that have a (small) radial outflow velocity that can mimic a stationary situation. In a small number of instances the material may actually be falling back on the star.

Acknowledgements. The authors wish to thank Drs J. Vink and P. Stee for the comments on an earlier version. This publication makes use of data products from the Two Micron All Sky Survey, which is a joint project of the University of Massachusetts and the Infra-red Processing and Analysis Center/California Institute of Technology, funded by the National Aeronautics and Space Administration and the National Science Foundation. This paper utilizes public domain data originally obtained by the MACHO Project, whose work was performed under the joint auspices of the US Department of Energy, National Nuclear Security Administration by the University of California, Lawrence Livermore National Laboratory under contract No. W-7405-Eng-48, the National Science Foundation through the Center for Particle Astrophysics of the University of California under cooperative agreement AST-8809616, and the 
Mount Stromlo and Siding Spring Observatory, part of the Australian National University.

\section{References}

Alcock, C., Akerloff, C. W., Allsman, R. A., et al. 1993, Nature, 365, 621 Apparao, K. M. V. 1991, ApJ, 376, 256

Aubourg, E., Bareyre, P., Brehin, S., et al. 1995, A\&A, 301, 1

Brussaard, P. J., \& van de Hulst, H. C. 1962, Rev. Mod. Phys., 34, 507

Clark, J. S., Tarasov, A. E., \& Panko, E. A. 2003, A\&A, 403, 239

Cook, K. H., Alcock, C., Allsman, H. A., et al. 1995, in Astrophysical Applications of Stellar Pulsation, IAU Colloq. 155, ASP Conf. Ser., 83, 221

Dachs, J., \& Wamsteker, W. 1982, A\&A, 107, 240

Dachs, J., Hanuschik, R., Kaiser, D., Ballereau, D., \& Bouchet, P. 1986, A\&AS, 63,87

de Wit, W. J., Beaulieu, J.-P., Lamers, H. J. G. L. M., Lesquoy, E., \& Marquette, J.-B. 2003, A\&A, 410, 199

Dougherty, S. M., Waters, L. B. F. M., Burki, G., et al. 1994, A\&A, 290, 609

Harmanec, P. 1998, A\&A, 334, 558

Hirata, R. 1982, in Be Stars, IAU Symp., 98, 41

Keller, S. C., Bessell, M. S., Cook, K. H., Geha, M., \& Syphers, D. 2002, AJ, 124,2039
Lamers, H. J. G. L. M., \& Waters, L. B. F. M. 1984, A\&A, 136, 37

Lamers, H. J. G. L. M., Beaulieu, J. P., \& De Wit, W. J. 1999, A\&A, 341, 827

Lee, U., Osaki, Y., \& Saio, H. 1991, MNRAS, 250, 432

Mennickent, R. E., \& Vogt, N. 1991, Rev. Mex. Astron. Astrofis., 22, 310

Mennickent, R. E., Pietrzyński, G., Gieren, W., \& Szewczyk, O. 2002, A\&A, 393,887

Mennickent, R. E., Pietrzynski, G., \& Gieren, W. 2003, in Interplay of Periodic, Cyclic and Stochastic Variability in Selected Areas of the H-R Diagram, ASP Conf. Ser., 292, 89

Meyssonnier, N., \& Azzopardi, M. 1993, A\&AS, 102, 451

Moujtahid, A., Zorec, J., \& Hubert, A. M. 1999, A\&A, 349, 151

Percy, J. R., \& Bakos, A. G. 2001, PASP, 113, 748

Poeckert, R., \& Marlborough, J. M. 1978, ApJS, 38, 229

Porter, J. M. 1999, A\&A, 348, 512

Rivinius, T., Baade, D., Stefl, S., et al. 1998, in Cyclical Variability in Stellar Winds, ed. L. Kaper \& A. W. Fullerton, 207

Rivinius, T., Baade, D., Štefl, S., \& Maintz, M. 2001, A\&A, 379, 257

Sabogal, B. E., Mennickent, R. E., Pietrzyński, G., \& Gieren, W. 2005, MNRAS, 361,1055

Skrutskie, M. F., Cutri, R. M., Stiening, R., et al. 2006, AJ, 131, 1163

Udalski, A., Kubiak, M., \& Szymanski, M. 1997, Acta Astron., 47, 319

Waters, L. B. F. M. 1986, A\&A, 162, 121 